\title{
Improving Fetal-maternal Outcome in Obstetric Anesthesia: Rhythm Disturbances and Vasopressor Use
}

\author{
Uma Hariharan $^{1 *}$ and Devang Bharati ${ }^{2}$ \\ ${ }^{1}$ Anesthesiology \& Intensive Care, Dr Ram Manohar Lohia Hospital \& Post Graduate Institute of Medical Education and Research, \\ New Delhi, India; ' Anesthesiology, Dr Ram Manohar Lohia Hospital \& Post Graduate Institute of Medical Education and Research, \\ New Delhi, India
}

\begin{abstract}
Regional anesthesia is the preferred modality for obstetric patients undergoing cesarean section due to the risks associated with general anesthesia in pregnant women. Single-shot spinal anesthesia is usually administered, which may be associated with post-block maternal hypotension, despite adequate intravenous fluid therapy. This is deleterious for the uteroplacental circulation, leading to poor outcomes in both the mother and fetus. There are several vasopressors which are used for treatment of these hypotensive episodes. Mephentermine is a sympathomimetic, having both direct and indirect actions. It has many side effects, however, including increased risks of arrhythmias, hypertension, central nervous system stimulation and abuse potential. Yet, it is still used in many developing countries, due to low cost and easy availability. We hereby report a case of rhythm disturbance with missed beats following use of mephentermine to counter hypotension after spinal anesthesia for cesarean section. The rhythm disturbance had a spontaneous resolution, with the patient maintaining hemodynamic stability during and after the episode.
\end{abstract}

Clinical commentary

A 30-year-old primigravida, $162 \mathrm{~cm}$ in height and weighing $84 \mathrm{~kg}$, was posted for emergency cesarean section at 39 weeks of gestation. Indication for caesarean section was fetal distress combined with nonprogress of labor. Routine preoperative clinical assessment was unremarkable, with hematological and biochemical investigations yielding results within normal limits. There was no history of any cardiac disease or any other co-morbidities.

The patient was taken to the Operation Theater table and electrocardiography, noninvasive blood pressure and pulse oximetry monitoring were started. The baseline heart rate was $92 \mathrm{bpm}$ and blood pressure was $110 / 76 \mathrm{mmHg}$ with oxygen saturation of $100 \%$. Widebore $(18 \mathrm{G})$ intravenous access was secured and the patient was preloaded with $500 \mathrm{~mL}$ of intravenous Ringer's lactate. Subarachnoid block was then administered under all aseptic precautions, with patient in the sitting position, in the $\mathrm{L}_{3}-\mathrm{L}_{4}$ intervertebral space using $25 \mathrm{G}$ Quincke's spinal needle. When free and clear flow of cerebrospinal fluid was obtained, bupivacaine hydrochloride (hyperbaric)

Keywords: Arrhythmias; Hypotension; Mephentermine Sulfate; Spinal anesthesia; Utero-placental circulation; Vasopressors.

Received: February 06, 2018; Revised: March 23, 2018; Accepted: April 13, 2018

${ }^{*}$ Correspondence to: Uma Hariharan, Anesthesiology \& Intensive Care, Dr Ram Manohar Lohia Hospital \& Post Graduate Institute of Medical Education and Research, BH 41, East Shalimar Bagh, Delhi 110088, India. Tel: +91-9811271093; Email: uma1708@gmail.com

How to cite this article: Hariharan U, Bharati D. Improving Fetal-maternal Outcome in Obstetric Anesthesia: Rhythm Disturbances and Vasopressor Use. Exploratory Research and Hypothesis in Medicine 2018;3(2):42-44. doi: 10.14218/ ERHM.2018.00001.
$0.5 \%$ at $11 \mathrm{mg}$ with fentanyl citrate at $10 \mathrm{mcg}$ (opioid) was injected into the subarachnoid space. Immediately after administering the spinal anesthesia, the patient was then turned to a supine position, with a wedge under the right hip. The blood pressure was recorded to be $100 / 72 \mathrm{mmHg}$ with a heart rate of $90 \mathrm{bpm}$.

After about $5 \mathrm{~m}$ postspinal anesthesia, blood pressure dropped to $74 / 50 \mathrm{mmHg}$, with heart rate of $84 \mathrm{bpm}$. Mephentermine hydrochloride $(6 \mathrm{mg})$ was administered intravenously and intravenous fluids were rushed to counter this hypotensive episode. The level of analgesia was assessed to be $\mathrm{T}_{6}$. Shortly thereafter, the patient's heart rate dropped to $40 \mathrm{bpm}$, with blood pressure of $76 / 54 \mathrm{mmHg}$ Immediately, $0.6 \mathrm{mg}$ of atropine was injected intravenously, with continued rushing-in of Ringer's lactate. The heart rate stabilized at $44 \mathrm{bpm}$, with a blood pressure of $76 / 52 \mathrm{mmHg}$. The level of analgesia was again assessed and was found to be $T_{4}$.

After $2 \mathrm{~m}$, there was sudden rise in heart rate to $94 \mathrm{bpm}$ and the blood pressure stabilized at $92 / 60 \mathrm{mmHg}$. This was accompanied by arrhythmias, with missed beats recorded on the electrocardiography monitor (Fig. 1). Since, by now the patient was hemodynamically stable $[\mathrm{BP}=114 / 80 \mathrm{~mm} \mathrm{Hg}, \mathrm{P}=74 / \mathrm{min}]$, no further interventions were undertaken, apart from vigilant monitoring, administration of intravenous fluids and gearing up of preparedness for any cardiovascular crisis. The surgery lasted for $1 \mathrm{~h}$, and one live, healthy male baby was delivered. Thereafter, the patient remained hemodynamically stable throughout the surgery, with heart rate varying from 86 bpm to $94 \mathrm{bpm}$ and mean arterial pressure remaining $>70 \mathrm{mmHg}$ at all times. After the surgery, the patient was transferred to the postanesthesia care unit and subsequently to a high-dependency unit for overnight observation.

Post-surgery cardiology work-up was carried out and yielded no remarkable findings. Electrocardiography (with long lead II) showed 


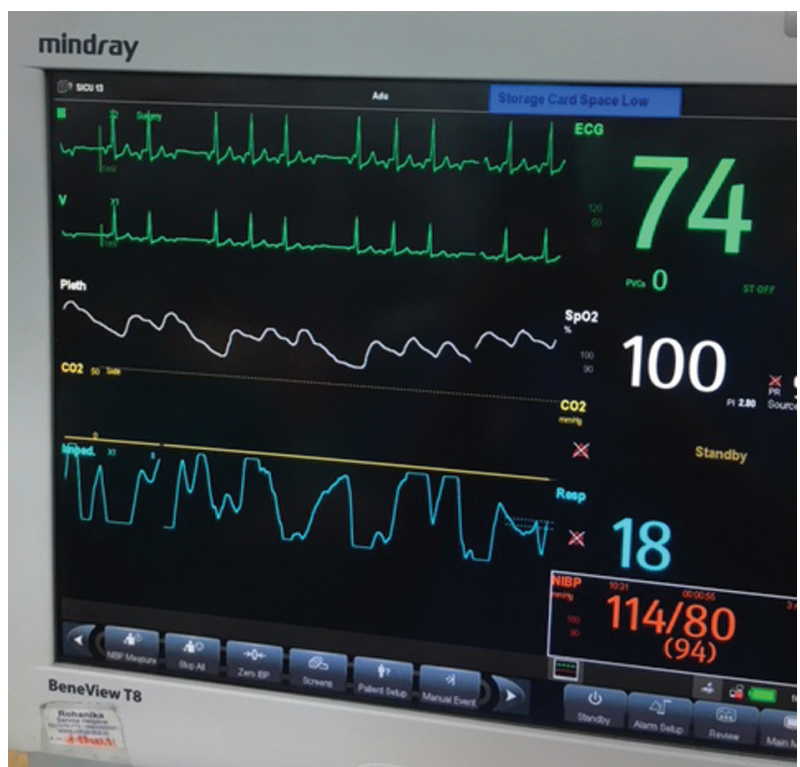

Fig. 1. ECG tracing showing rhythm disturbances following mephentermine for hypotension.

normal sinus rhythm, with a rate of $82 \mathrm{bpm}$. Echocardiograph showed normal-sized cardiac chambers with no regional wall motion abnormality, global ejection fraction of $60 \%$, and no diastolic or systolic dysfunction. Valves were structurally normal and no other intracardiac defect, intracardiac mass or pericardial effusion was seen. The 24-h Holter monitoring was normal. Regular follow-up was advised with the obstetrician and the cardiologist at the time of discharge.

There have been several case reports on hypotension and bradycardia following spinal anesthesia in the obstetric population. ${ }^{1}$ Bupivacaine (cardiac toxicity) is implicated in this regard especially. Ropivacaine offers better hemodynamic stability than bupivacaine but plain ropivacaine has also been associated with an increased failure rate, $, 2,3$ and that is why bupivacaine continues to be the preferred agent. The occurrence of arrhythmias with mephentermine, especially with missed beats is uncommon.

The patient presented herein had hypotension and bradycardia preceding the rhythm disturbance. Vagal stimulation during uterine manipulation could be a possible cause. ${ }^{4}$ The more likely cause of rhythm disturbance in this patient could be the use of intravenous mephentermine sulfate..$^{5}$ It is a synthetic catecholamine, vasoconstrictor inotropic agent (mixed alpha and beta receptor agonist activity with both direct and indirect effects). It is a sympathomimetic, capable of stimulating alpha and beta-adrenergic receptors (direct action). It also acts indirectly by releasing norepinephrine from neuronal storage sites.

Mephentermine is known to cause central nervous system excitation, increased uterine contractions, rapid tachyphylaxis and potentiate the risk of arrhythmias. ${ }^{6}$ Other safer vasopressors of use in obstetric anesthesia include ephedrine (causing both direct alpha- and beta-agonistic action and more prominent indirect action due to release of norepinephrine from sympathetic neurons) and phenylephrine hydrochloride (selective alpha-1 receptor agonistic action and beta-agonist action only at high doses).

Though ephedrine has been purported to be safer for use in pregnant woman, ${ }^{7}$ current guidelines support the use of phenylephrine, ${ }^{8}$ especially prophylactic infusion to prevent hypotension after spinal anesthesia during cesarean section. Nonetheless, mephentermine continues to be used in the developing world due to multiple reasons. First is its low cost. ${ }^{9}$ Second, is its comparability in efficacy to both phenylephrine and ephedrine. ${ }^{10}$ And, third, is that unlike phenylephrine it does not require serial dilutions.

Adequate fluid therapy, prevention of supine-hypotension syndrome and avoiding cephalad spread of spinal block can minimize the chances of maternal hypotension and, hence, aid in maintaining the uteroplacental perfusion. In addition, the use of mephentermine should be minimized, if possible, to reduce the risk of rhythm disturbances and other adverse events. There are dynamic trends in recent times for fluid management and vasopressor therapy in the prevention and treatment of hypotension following neuraxial blockade during cesarean section. ${ }^{11}$

The electrocardiography changes in our patient had a spontaneous resolution (without any treatment) and the patient remained hemodynamically stable. Rhythm disturbances following obstetric spinal anesthesia have multifactorial causative factors and prevention of severe hypotension should be our paramount goal. Future randomized controlled trials are required to standardize protocols for preventive and treatment strategies regarding maternal hypotension and rhythm disturbances. Obstetric anesthesiologists must take extra care in the operation theatre for preparedness to detect such situations early, handle them safely and strive to improve fetal-maternal outcome.

\section{Future prospects}

The choice of vasopressor for perioperative obstetric hypotension is still debatable. Current studies favor the use of phenylephrine, especially in elective cesarean sections, due to better fetal acidbase status. However, this cannot be extrapolated to emergency obstetric surgeries, which may be compounded with preexisting fetal acidosis and maternal cardiorespiratory compromise. Moreover, our case scenario pertains to cardiac rhythm disturbances, most likely following mephentermine administration for correction of maternal hypotension. Future research must be directed in this path of developing an ideal vasopressor agent for obstetric anesthesia, especially in emergency circumstances. Standard and uniform guidelines must be developed for prevention and treatment of such cardiac events, for a successful fetal-maternal outcome.

\section{Acknowledgments}

The patient provided informed consent for publication of this data. No sources of funding were used for this study.

\section{Conflict of interest}

The authors have no conflict of interests related to this publication.

\section{Author contributions}

Conceived, wrote, edited and corrected the entire manuscript in all stages, including correspondence (UH), collected case details (DB), prepared rough script (DB), obtained review of literature (DB).

\section{References}

[1] Brenck F, Hartmann B, Katzer C, Obaid R, Bruggmann D, Benson M, et 
al. Hypotension after spinal anesthesia for cesarean section: indentification of risk factors using an anesthesia information management system. J Clin Monit Comput 2009;23(2):85-92. doi:10.1007/s10877009-9168-x.

[2] Erturk E, Tutuncu C, Eroglu A, Gokben M. Clinical comparison of 12 $\mathrm{mg}$ ropivacaine and $8 \mathrm{mg}$ bupivacaine, both with $20 \mu \mathrm{g}$ fentanyl, in spinal anaesthesia for major orthopaedic surgery in geriatric patients. Med Princ Pract 2010;19(2):142-147. doi:10.1159/000249581.

[3] Malinovsky JM, Charles F, Kick O, Lepage JY, Malinge M, Cozian A, et al. Intrathecal anesthesia: ropivacaine versus bupivacaine. Anesth Analg 2000;91(6):1457-1460. doi:10.1097/00000539-20001200000030.

[4] Jang YE, Do SH, Song IA. Vasovagal cardiac arrest during spinal anesthesisa for Cesarean section - A case report. Korean J Anesthesiol 2013;64(1):77-81. doi:10.4097/kjae.2013.64.1.77.

[5] Hingson RA, Davis HS, Inman CE, Lelievre RE. A clinical evaluation of mephentermine as a vasopressor in surgery and obstetrics. Am Pract Dig Treat 1955;6(7):1004-1014.

[6] Mohta M, Agarwal D, Gupta LK, Sethi AK, Tyagi A. Potency of mephentermine for prevention of post-spinal hypotension. Anaesth Intensive Care 2009;37(4):568-570.
[7] Nag DS, Samaddar DP, Chatterjee A, Kumar H, Dembla A. Vasopressors in obstetric anesthesia: A current perspective. World J Clin Cases 2015;3(1):58-64. doi:10.12998/wjcc.v3.i1.58.

[8] Kinsella SM, Carvalho B, Dyer RA, Fernando R, McDonnell N, Mercier FJ, et al. International consensus statement on the management of hypotension with vasopressors during cesarean section under spinal anesthesia. Anaesthesia 2018;73(1):71-92. doi:10.1111/ anae.14080.

[9] Mohta M, Janani SS, Sethi AK, Agarwal D, Tyagi A. Comparison of phenylephrine hydrochloride and mephentermine sulphate for prevention of post spinal hypotension. Anaesthesia 2010;65(12):12001205. doi:10.1111/j.1365-2044.2010.06559.x.

[10] Dua D, Jadliwala R, Gondalia D, Parmar V, Jain A. Comparison of bolus phenylephrine, ephedrine and mephentermine for maintenance of arterial pressure during spinal anaesthesia in caesarean section. Int J Pharm Sci Res 2014;5(6):2412-2417. doi:10.13040/ IJPSR.0975-8232.5(6).2412-17.

[11] Mitra JK, Roy J, Bhattacharyya P, Yunus M, Lyngdoh NM. Changing trends in the management of hypotension following spinal anesthesia in cesarean section. J Postgrad Med 2013;59(2):121-126. doi:10.4103/0022-3859.113840. 